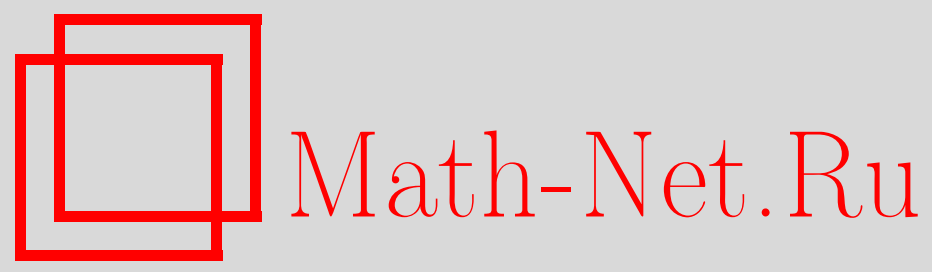

А. Л. Скубачевский, Р. В. Шамин, Первая смешанная задача для параболического дифференциально-разностного уравнения, Матем. заметки, 1999, том 66, выпуск 1, 145153

DOI: https://doi.org/10.4213/mzm1149

Использование Общероссийского математического портала Math-Net.Ru подразумевает, что вы прочитали и согласны с пользовательским соглашением http://www.mathnet.ru/rus/agreement

Параметры загрузки:

IP: 18.234 .156 .22

26 апреля 2023 г., 14:34:01

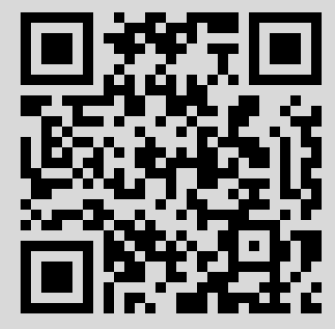




\title{
ПЕРВАЯ СМЕШАННАЯ ЗАДАЧА ДЛЯ ПАРАБОЛИЧЕСКОГО ДИФФЕРЕНЦИАЛЬНО-РАЗНОСТНОГО УРАВНЕНИЯ
}

\author{
А. Л. Скубачевский, Р. В. Шамин
}

\begin{abstract}
Рассматривается первая смешанная задача для параболического дифференциально-разностного уравнения со сдвигами по пространственным переменным. Доказаны однозначная разрешимость этой задачи и гладкость обобшенных решений в некоторых цилиндрических подобластях. Показано, что гладкость обобщенных решений может нарушаться на границах соседних подобластей.
\end{abstract}

Библиография: 7 названий.

Введение. Параболические функционально-дифференциальные уравнения возникают при исследовании нелинейных оптических систем с двумерной обратной связью [1]-[3]. В отличие от параболических дифференциальных уравнений эти уравнения обладают рядом принципиально новых свойств. Например, гладкость обобщенных решений может нарушаться внутри цилиндрической области даже при бесконечно гладкой правой части уравнения.

В настоящей работе рассматривается первая смешанная задача для параболического дифференциально-разностного уравнения со сдвигами по пространственным переменным. Доказаны однозначная разрешимость этой задачи и гладкость обобшенных решений в некоторых цилиндрических подобластях. Показано также, что гладкость обобщенных решений может нарушаться на границах соседних подобластей.

1. Постановка задачи. Пусть $Q \subset \mathbb{R}^{n}$ - ограниченная область с границей $\partial Q=$ $\bigcup_{i} \overline{M_{i}}\left(i=1, \ldots, N_{\circ}\right)$, где $M_{i}-(n-1)$-мерные многообразия класса $C^{\infty}$, которые являются открытыми и связньми в топологии $\partial Q$. Пусть в окрестности каждой точки $g \in \partial Q \backslash \bigcup_{i} M_{i}$ область $Q$ - диффеоморфна $n$-мерному двугранному углу, если $n \geqslant 3$, и плоскому углу, если $n=2$.

Мы будем обозначать через $W_{2}^{k}(Q)$ пространство Соболева комплекснозначных функций из $L_{2}(Q)$, имеюших все обобщенные производные вплоть до $k$-го порядка из $L_{2}(Q)$, с нормой

$$
\|u\|_{W_{2}^{k}(Q)}=\left\{\sum_{|\alpha| \leqslant k} \int_{Q}\left|\mathcal{D}^{\alpha} u(x)\right|^{2} d x\right\}^{1 / 2} .
$$

Работа выполнена при финансовой поддержке гранта Конкурсного Центра по фундаментальным и естественным наукам при Санкт-Петербургском Государственном университете (№ 97-14.3-37). 
Через $\stackrel{\circ}{W}_{2}^{k}(Q)$ обозначим замыкание множества финитных бесконечно дифференцируемых функций $\dot{C}^{\infty}(Q)$ в $W_{2}^{k}(Q)$, а через $W_{2}^{-1}(Q)$ обозначим пространство, сопряженное к $\stackrel{\circ}{W} \frac{1}{2}(Q)$.

Введем ограниченньй дифференциально-разностный оператор $A_{R}: \stackrel{\circ}{W_{2}^{1}}(Q) \rightarrow$ $W_{2}^{-1}(Q)$ по формуле

$$
A_{R} u=\sum_{i, j=1}^{n}\left(R_{i j Q} u_{x_{j}}\right)_{x_{i}}+\sum_{i=1}^{n} R_{i Q} u_{x_{i}}+R_{0 Q} u .
$$

Здесь $R_{i j Q}=P_{Q} R_{i j} I_{Q}, R_{i Q}=P_{Q} R_{i} I_{Q}$,

$$
\begin{aligned}
R_{i j} u(x) & =\sum_{h \in M} a_{i j h}(x) u(x+h) \quad(i, j=1, \ldots, h), \\
R_{i} u(x) & =\sum_{h \in M} a_{i h}(x) u(x+h) \quad(i=0,1, \ldots, n),
\end{aligned}
$$

$M \subset \mathbb{R}^{n}$ - конечное множество векторов с целочисленными координатами, $a_{i j h}, a_{i h} \in$ $C^{\infty}(\bar{Q}) ; \quad I_{Q}: L_{2}(Q) \rightarrow L_{2}\left(\mathbb{R}^{n}\right)$ - оператор продолжения функции из $L_{2}(Q)$ нулем в $\mathbb{R}^{n} \backslash Q ; P_{Q}: L_{2}\left(\mathbb{R}^{n}\right) \rightarrow L_{2}(Q)$ - оператор сужения функции из $L_{2}\left(\mathbb{R}^{n}\right)$ на $Q$.

ОПРЕДЕЛЕНИЕ 1. Оператор $-A_{R}$ мы будем назьвать сильно әллиптическим, если сушествуют константы $c_{1}>0$ и $c_{2} \geqslant 0$ такие, что для всех $u \in \dot{C}^{\infty}(Q)$

$$
-\operatorname{Re}\left(A_{R} u, u\right)_{L_{2}(Q)} \geqslant c_{1}\|u\|_{W_{2}^{1}(Q)}^{2}-c_{2}\|u\|_{L_{2}(Q)}^{2} .
$$

Необходимые и достаточные условия сильной эллиптичности в алгебраической форме будут сформулированы в конще этого пункта.

Рассмотрим дифференциально-разностное уравнение

$$
u_{t}(x, t)-A_{R} u(x, t)=f(x, t) \quad\left((x, t) \in \Omega_{T}\right)
$$

с краевым условием

$$
\left.u\right|_{\Gamma_{T}}=0 \quad\left((x, t) \in \Gamma_{T}\right)
$$

и начальным условием

$$
\left.u\right|_{t=0}=\varphi(x) \quad(x \in Q)
$$

где $\Omega_{T}=Q \times(0, T), \Gamma_{T}=\partial Q \times(0, T), 0<T<\infty$.

Всюду в дальнейшем мы будем предполагать, что оператор $-A_{R}-$ сильно эллиптический. В этом случае задачу (1.3)-(1.5) естественно назьвать первой смешанной задачей для параболического дифференциально-разностного уравнения. Не ограничивая обшности, мы будем считать, что в неравенстве $(1.2) c_{2}=0$. Действительно, стандартная замена неизвестной функции $u=\exp \left(c_{2} t\right) w$ сводит уравнение $(1.3)$ к виду $\left(-A_{R}+\right.$ $\left.c_{2} I\right) w+w_{t}=\exp \left(-c_{2} t\right) f(x, t) \quad\left((x, t) \in \Omega_{T}\right)$.

Для того чтобы сформулировать условия сильной эллиптичности оператора $-A_{R}$, введем некоторые вспомогательные обозначения. Эти обозначения понадобятся нам также в п. 4 для исследования гладкости обобщенных решений задачи (1.3)-(1.5). Обозначим через $G$ аддитивную абелеву группу, порожденную множеством $M$, а через $Q_{r}$ открытые связные компоненты множества

$$
Q \backslash\left(\bigcup_{h \in G}(\partial Q+h)\right) .
$$


ОПРЕДЕЛЕниЕ 2 . Множества $Q_{r}$ мы будем называть $\mathcal{R}$ всевозможных подобластей $Q_{r}-$ разбиением множества $Q$.

Разбиение $\mathcal{R}$ естественным образом распадается на непересекающиеся классы: будем считать, что подобласти $Q_{r_{1}}, Q_{r_{2}} \in \mathcal{R}$ принадлежат одному классу, если существует $h \in G$ такое, что $Q_{r_{2}}=Q_{r_{1}}+h$. Обозначим подобласти $Q_{r}$ через $Q_{s l}$, где $s-$ номер класса $(s=1,2, \ldots)$, a $l$ - порядковьй номер подобласти в $s$-м классе. В силу ограниченности области $Q$ каждый класс состоит из конечного числа $N=N(s)$ подобластей $Q_{s l}$ и $N(s) \leqslant([\operatorname{diam} Q]+1)^{n}$.

Для того чтобы сформулировать необходимые условия сильной эллиптичности в алгебраической форме, мы введем матрищы $R_{i j s}(x)\left(x \in \bar{Q}_{s 1}\right)$ порядка $N(s) \times N(s)$ с элементами

$$
r_{k l}^{i j s}(x)= \begin{cases}a_{i j h}\left(x+h_{s k}\right) & \left(h=h_{s l}-h_{s k} \in M\right), \\ 0 & \left(h_{s l}-h_{s k} \notin M\right) .\end{cases}
$$

В силу теоремы 9.1 из [3] если оператор $-A_{R}$ сильно эллиптический, то для всех $s=1,2, \ldots, x \in \bar{Q}_{s 1}$ и $0 \neq \xi \in \mathbb{R}^{n}$ матрицы

$$
\sum_{i, j=1}^{n}\left(R_{i j s}(x)+R_{i j s}^{*}(x)\right) \xi_{i} \xi_{j}
$$

положительно определены.

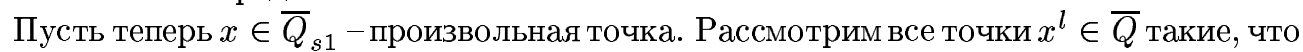
$x^{l}-x \in G$. Поскольку область $Q$ ограниченная, множество $\left\{x^{l}\right\}$ состоит из конечного числа точек $I=I(s, x)(I \geqslant N(s))$. Перенумеруем точки $x^{l}$ так, что $x^{l}=x+h_{s l}$ для $l=1, \ldots, N=N(s), x^{1}=x$, где $h_{s l}$ удовлетворяет условию $Q_{s l}=Q_{s 1}+h_{s l}$. Введем матрицы $A_{i j s}(x)$ порядка $I \times I$ с элементами $a_{l k}^{i j s}(x)$ по формуле

$$
a_{l k}^{i j s}(x)= \begin{cases}a_{i j h}\left(x^{l}\right) & \left(h=x^{k}-x^{l} \in M\right), \\ 0 & \left(x^{k}-x^{l} \notin M\right) .\end{cases}
$$

В силу теоремы 9.2, из [3] если для всех $s=1,2, \ldots, x \in \bar{Q}_{s 1}$ и $0 \neq \xi \in \mathbb{R}^{n}$ матрицы

$$
\sum_{i, j=1}^{n}\left(A_{i j s}(x)+A_{i j s}^{*}(x)\right) \xi_{i} \xi_{j}
$$

положительно определены, то оператор $-A_{R}$ - сильно эллиптический.

Очевидно, если $I=N$, то матрица $R_{i j s}(x)$ равна матрице $A_{i j s}(x)$. Если $N<I$, то матрица $R_{i j s}(x)$ получается из матрицы $A_{i j s}$ вычеркиванием последних $I-N$ строк и столбцов.

2. Существование и единственность обобщенного решения. Обозначим через $W_{2}^{k, 0}\left(\Omega_{T}\right)$ пространство Соболева комплекснозначных функций $u \in L_{2}\left(\Omega_{T}\right)$, имеющих обобщенные производные $u_{x_{i}} \in L_{2}\left(\Omega_{T}\right)(i=1, \ldots, n)$ с нормой

$$
\|u\|_{W_{2}^{k, 0}\left(\Omega_{T}\right)}=\left\{\sum_{|\alpha| \leqslant k} \int_{\Omega_{T}}\left|\mathcal{D}^{\alpha} u(x, t)\right|^{2} d x d t+\int_{\Omega_{T}}|u(x, t)|^{2} d x d t\right\}^{1 / 2} .
$$


Положим $\mathcal{V}=L_{2}\left((0, T) ; \stackrel{\circ}{W} \frac{1}{2}(Q)\right)$. Легко видеть, что $\mathcal{V}=\left\{v \in W_{2}^{1,0}\left(\Omega_{T}\right):\left.v\right|_{\Gamma_{T}}=\right.$ $0\}$, а сопряженное пространство имеет вид $\mathcal{V}^{\prime}=L_{2}\left((0, T) ; W_{2}^{-1}(Q)\right)$. Введем ограниченньй оператор $L_{R}: \mathcal{V} \rightarrow \mathcal{V}^{\prime}$ по формуле $L_{R} v(\cdot, t)=-A_{R} v(\cdot, t)$ для почти всех $t \in(0, T)$.

Введем также неограниченньй оператор $\Lambda_{t}: \mathcal{V}^{\prime} \supset D\left(\Lambda_{t}\right) \rightarrow \mathcal{V}^{\prime}$, действующий в смысле распределений со значениями в $\mathcal{V}^{\prime}$ по формуле $\Lambda_{t} v(\cdot, t)=\partial v(\cdot, t) / \partial t$ с областью определения $D\left(\Lambda_{t}\right)=\left\{v \in \mathcal{V}^{\prime}: \Lambda_{t} v \in \mathcal{V}^{\prime}\right\}$.

Отметим, что если функция $u \in \mathcal{V}$ удовлетворяет операторному уравнению

$$
\Lambda_{t} u+L_{R} u=f
$$

где $f \in \mathcal{V}^{\prime}$, то в силу теоремы 3.1 и предложения 2.1 из $\left[4\right.$, гл. 1] $u \in C^{0}\left([0, T] ; L_{2}(Q)\right)$. Поэтому $\left.u\right|_{t=0}$ имеет смысл.

Пусть $f \in \mathcal{V}^{\prime}, \varphi \in L_{2}(Q)$.

ОПРЕДЕЛЕНИЕ 3. Будем назьвать функцию $u \in \mathcal{V} \cap D\left(\Lambda_{t}\right)$ обобщенным решением задачи (1.3)-(1.5), если она удовлетворяет операторному уравнению (2.1) и начальному условию (1.5).

ТЕОремА 1. Пусть дифференциально-разностный оператор $-A_{R}-$ сильно әллиптический. Тогда для любых $f \in \mathcal{V}^{\prime} u \varphi \in L_{2}(Q)$ задача (1.3)-(1.5) имеет единственное обобщенное решение $u \in \mathcal{V} \cap D\left(\Lambda_{t}\right)$.

ДокАЗАТЕЛЬСтво. Очевидно, разностные операторы $R_{i j Q}: L_{2}(Q) \rightarrow L_{2}(Q)$ являются ограниченными. Следовательно, существует константа $c_{0}>0$ такая, что

$$
\left|\left(A_{R} u, v\right)_{L_{2}(Q)}\right| \leqslant c_{0}\|u\|_{W_{2}^{1}(Q)}\|v\|_{W_{2}^{1}(Q)}
$$

для любых $u, v \in \dot{C}^{\infty}(Q)$. Из неравенств $(1.2),(2.2)$ данной работы, а также из теоремы 4.1 и замечания 4.3 [4, гл. 3] вытекает существование и единственность обобщенного решения задачи (1.3)-(1.5).

Предположим теперь, что $f \in L_{2}\left(\Omega_{T}\right), \varphi \in L_{2}(Q)$. В этом случае мы дадим определение обобщенного решения задачи (1.3)-(1.5) в смысле интегрального тождества.

ОПРЕДЕЛЕНИЕ 4. Мы будем назьвать функцию $u \in \mathcal{V}$ обобщенным решением задачи (1.3)-(1.5), если для любых $v \in\left\{v \in W_{2}^{1}\left(\Omega_{T}\right):\left.v\right|_{\Gamma_{T}}=0,\left.v\right|_{t=T}=0\right\}$ выполняется интегральное тождество

$$
\int_{\Omega_{T}}\left(-u \bar{v}_{t}+\sum_{i, j=1}^{n} R_{i j Q} u_{x_{j}} \bar{v}_{x_{i}}\right) d x d t=\int_{\Omega_{T}} f \bar{v} d x d t+\left.\int_{Q} \varphi \bar{v}\right|_{t=0} d x
$$

ТЕОрема 2. Пусть оператор $-A_{R}$ - сильно әллиптический и пусть $f \in L_{2}\left(\Omega_{T}\right)$, $\varphi \in L_{2}(Q)$. Тогда определения 3 и 4 әквивалентны. 
ДокАЗАТЕльСтво. Эквивалентность определений 3,4 будет следовать из теорем $4.1,4.2,\left[4\right.$, гл. 3], если показать, что множество $\mathcal{V}_{1}=\left\{v \in W_{2}^{1}\left(\Omega_{T}\right):\left.v\right|_{\Gamma_{T}}=0,\left.v\right|_{t=T}=\right.$ $0\}$ всюду плотно в пространстве $\mathcal{V}_{2}=\left\{v \in \mathcal{V} \cap D\left(\Lambda_{t}\right):\left.v\right|_{t=T}=0\right\}$.

Действительно, пусть $v \in \mathcal{V}_{2}$. Рассмотрим последовательность вещественнозначных функций $\xi_{n} \in C^{\infty}[0, T]$ таких, что $\xi_{n}(t)=1(0 \leqslant t \leqslant T-2 / n), \quad \xi_{n}(t)=0$ $(T-1 / n \leqslant t \leqslant T) ; 0 \leqslant \xi_{n}(t) \leqslant 1,\left|\xi_{n}^{\prime}(t)\right| \leqslant C n(0 \leqslant t \leqslant T)$. Очевидно, $\xi_{n} v \rightarrow v$ при $n \rightarrow \infty$ в пространстве $\mathcal{V}_{2}$. Сглаживая функции $\xi_{n} v$ по $t$, получим последовательность функций $v_{n}(t)$ со значениями в $\stackrel{\circ}{W} \frac{1}{2}(Q)$, которые бесконечно дифференцируемы по $t$ и имеют носители на $[0, T)$. По построению $v_{n} \rightarrow v$ в пространстве $\mathcal{V}_{2}$, при этом $v_{n} \in \mathcal{V}_{1}$.

\section{3. Аналитические полугруппы.}

ОПРЕДЕЛЕниЕ 5 . Пусть $X$ - банахово пространство. Однопараметрическое семейство линейных ограниченных операторов $T_{t}: X \rightarrow X(t \geqslant 0)$ называется сильно непрерывной полугруппой или $C_{0}$-полугруппой, если

1) $T_{0}=I$

2) $T_{t+s}=T_{t} T_{s}(t, s \geqslant 0)$;

3) $\lim _{t \searrow 0} T_{t} x=x$ (для любого $\left.x \in X\right)$.

ОПРЕДЕЛЕНИЕ 6 . Полугрупа класса $C_{0}$ назьвается сжимающей, если $\left\|T_{t}\right\| \leqslant 1$ $(t \geqslant 0)$.

ОПРЕДЕЛЕНИЕ 7. Линейный оператор $A: X \supset D(A) \rightarrow X$, определенный по формуле

$$
A x=\lim _{t \searrow 0} \frac{T_{t} x-x}{t}\left(x \in D(A)=\left\{x \in X: \lim _{t \searrow_{0}} \frac{T_{t} x-x}{t} \text { сушествует }\right\}\right)
$$

назьвается инфинитезимальным производящим оператором сильно непрерывной nолугруппы $\left\{T_{t}\right\}$.

Введем неограниченньй оператор $\mathcal{A}_{R}: L_{2}(Q) \supset D\left(\mathcal{A}_{R}\right) \rightarrow L_{2}(Q)$, действующий в пространстве распределений $D^{\prime}(Q)$ по формуле $\mathcal{A}_{R} u=A_{R} u\left(u \in D\left(\mathcal{A}_{R}\right)=\{u \in\right.$ $\left.\left.\stackrel{\circ}{W} \frac{1}{2}(Q): \mathcal{A}_{R} u \in L_{2}(Q)\right\}\right)$.

ТеОрема 3. Пусть оператор $-A_{R}-$ сильно әллиптический. Тогда оператор $\mathcal{A}_{R}$ является инфинитезимальным производящим оператором сжимающей полуzруппь $T_{t}$ в $L_{2}(Q)$.

ДокАЗАТЕЛЬСТВО. Из неравенства (1.2) с константой $c_{2}=0$ и теоремы 10.1 из [5] следует, что оператор $\mathcal{A}_{R}$ - замкнутьй, а спектр $\sigma\left(\mathcal{A}_{R}\right) \subset\{\lambda \in \mathbb{C}: \operatorname{Re} \lambda<0\}$, при этом для любых $u \in D\left(\mathcal{A}_{R}\right)$

$$
-\operatorname{Re}\left(\mathcal{A}_{R} u, u\right)_{L_{2}(Q)} \geqslant c_{1}\|u\|_{W_{2}^{1}(Q)}^{2} .
$$

Из (3.1) и неравенства Коши-Буняковского следует, что для $u \in D\left(\mathcal{A}_{R}\right)$ и $\lambda>0$

Таким образом,

$$
\left\|\left(\lambda I-\mathcal{A}_{R}\right) u\right\|_{L_{2}(Q)}\|u\|_{L_{2}(Q)} \geqslant \lambda\|u\|_{L_{2}(Q)}^{2} .
$$

$$
\left\|R\left(\lambda, \mathcal{A}_{R}\right)\right\| \leqslant \frac{1}{\lambda}
$$

Поэтому в силу теоремы Хилле-Иосиды (см., $[6, \S 1.3])$ оператор $\mathcal{A}_{R}$ является инфинитезимальным производящим оператором сжимающей полугруппы $T_{t}$ в $L_{2}(Q)$.

Обозначим $\Delta=\left\{z \in \mathbb{C}: \varphi_{1}<\arg z<\varphi_{2}\right\}$, где $\varphi_{1}<0<\varphi_{2}$. 
ОПРЕДЕЛЕНИЕ 8. Однопараметрическое семейство линейных ограниченных операторов $T_{z}: X \rightarrow X(z \in \Delta)$ назьвается аналитической полугруппой в $\Delta$, если

1) функция $z \rightarrow T_{z}$ является аналитической в $\Delta$;

2) $T_{0}=I$ и $\lim _{z \rightarrow 0, z \in \Delta} T_{z} x=x$ (для любого $x \in X$ );

3) $T_{z_{1}+z_{2}}=T_{z_{1}} T_{z_{2}}$ (для любых $z_{1}, z_{2} \in \Delta$ ).

Полугруппа $T_{t}$ называется аналитической, если она аналитическая в некотором секторе $\Delta$, содержащем положительную вещественную полуось.

ТЕОремА 4. Пусть оператор $-A_{R}$ - сильно әллиптический и, следовательно, оператор $\mathcal{A}_{R}$ является инфинитезимальным производящим оператором сжимающей полугруппь $T_{t}$ в $L_{2}(Q)$. Тогда полугруппу $T_{t}$ можно продолжить до аналитической полугруппь $T_{z}$ в некотором секторе $\Delta_{\delta}=\{z \in \mathbb{C}:|\arg z|<\delta\}$.

ДоКАЗАТЕЛЬСТВО проводится стандартными методами, основанными на использовании оценки нормы резольвенты инфинитезимального производящего оператора полугруппы. В свою очередь, указанная оценка вьводится из неравенства типа Гординга (1.2) (см. теорему 2.7 из [6, § 7.2] или теорему 1 из, [7, гл. $14, \S 1])$.

\section{4. Гладкость обобщенных решений.}

ОПРЕДЕЛЕНИЕ 9. Обобщенное решение задачи (1.3)-(1.5) при $f \in L_{2}\left(\Omega_{T}\right)$ и $\varphi \in$ $L_{2}(Q)$ мы будем называть сильным решением, если $u_{t} \in L_{2}\left(\Omega_{T}\right)$.

Для исследования вопроса о гладкости обобщенных решений задачи (1.3)-(1.5) введем некоторые дополнительные обозначения. Рассмотрим множества

$$
\mathcal{K}=\bigcup_{h_{1}, h_{2} \in G}\left\{\bar{Q} \cap\left(\partial Q+h_{1}\right) \cap \overline{\left[\left(\partial Q+h_{2}\right) \backslash\left(\partial Q+h_{1}\right)\right]}\right\},
$$

а также

$$
\mathcal{K}^{\varepsilon}=\left\{x \in \mathbb{R}^{n}: \rho(x, \mathcal{K})<\varepsilon\right\}
$$

где $\varepsilon>0$.

Пусть $D \subset \mathbb{R}^{n}$ - ограниченная область. Обозначим через $W_{2}^{2 k, k}(D \times(0, T))$ пространство Соболева комплекснозначных функций из $L_{2}(D \times(0, T))$, имеющих все обобщенные производные $\mathcal{D}_{x}^{\alpha} \mathcal{D}_{t}^{\beta} u \in L_{2}(D \times(0, T))(|\alpha|+2 \beta \leqslant 2 k)$, с нормой

$$
\|u\|_{W_{2}^{2 k, k}(D \times(0, T))}=\left\{\sum_{|\alpha|+2 \beta \leqslant 2 k} \int_{\Omega_{T}}\left|\mathcal{D}_{x}^{\alpha} \mathcal{D}_{t}^{\beta} u(x, t)\right|^{2} d x d t\right\}^{1 / 2} .
$$

ТеОрема 5. Пусть $\partial Q \backslash \bigcup_{i} M_{i} \subset \mathcal{K}$ и пусть дифференииально-разностный оператор $-A_{R}-$ сильно әллиптический. Тогда для любых $\varphi \in D\left(\mathcal{A}_{R}\right)$ u $f \in L_{2}\left(\Omega_{T}\right)$ таких, что $f_{t} \in L_{2}\left(\Omega_{T}\right)$, задача (1.3)-(1.5) имеет единственное сильное речение, которое определяется по формуле

$$
u(x, t)=T_{t} \varphi(x)+\int_{0}^{t} T_{t-s} f(x, s) d s .
$$

Более того, для каждого $\varepsilon>0$ и всех $s=1,2, \ldots ; \quad l=1, \ldots, N(s) \quad u(x, t) \in$ $W_{2}^{2,1}\left(\left(Q_{s l} \backslash \mathcal{K}^{\varepsilon}\right) \times(0, T)\right)$. 
ДокАЗАтЕльство. Существование и единственность решения задачи (1.3)-(1.5) и формула (4.1) следуют из теоремы 3 настоящей работы и следствия 2.10 из [6, $§ 4.2]$. Из определения сильного решения и уравнения (1.3) следует, что

$$
-A_{R} u(\cdot, t)=F(\cdot, t)
$$

где $F(\cdot, t)=f(\cdot, t)-u_{t}(\cdot, t) \in L_{2}(Q)$ для почти всех $t \in(0, T)$. В силу теоремы 11.2 из [5] о гладкости обобщенных решений краевых задач для сильно эллиптических дифференциально-разностных уравнений для любого $\varepsilon>0$

$$
u(\cdot, t) \in W_{2}^{2}\left(Q_{s l} \backslash \mathcal{K}^{\varepsilon}\right)
$$

И

$$
\|u\|_{W_{2}^{2}\left(Q_{s l} \backslash \mathcal{K}^{\varepsilon}\right)} \leqslant c\|F\|_{L_{2}(Q)}
$$

для почти всех $t \in(0, T)$ и $s=1,2, \ldots ; l=1, \ldots, N(s)$, где $c>0$ не зависит от $t$.

Возводя обе части неравенства (4.3) в квадрат и интегрируя от 0 до $T$, получим

$$
\|u\|_{W_{2}^{2,0}\left(\left(Q_{s l} \backslash \mathcal{K}^{\varepsilon}\right) \times(0, T)\right)} \leqslant c_{1}\left(\|f\|_{L_{2}\left(\Omega_{T}\right)}+\left\|u_{t}\right\|_{L_{2}\left(\Omega_{T}\right)}\right) .
$$

Отсюда следует, что $u \in W_{2}^{2,1}\left(\left(Q_{s l} \backslash \mathcal{K}^{\varepsilon}\right) \times(0, T)\right)$.

ЗАмЕчАниЕ 1. При выполнении условий теоремы 5 в силу теорем 1,5 обобщенное решение задачи (1.3)-(1.5) является сильньм решением и, следовательно, принадлежит пространству $W_{2}^{2,1}\left(\left(Q_{s l} \backslash \mathcal{K}^{\varepsilon}\right) \times(0, T)\right)$.

Как показывает следуюший пример, гладкость сильных решений задачи (1.3)-(1.5) может нарушаться на границе соседних цилиндров $Q_{s_{1} l_{1}} \times(0, T)$ и $Q_{s_{2} l_{2}} \times(0, T)$, а также вблизи множества $\mathcal{K} \times(0, T)$.

ПРимеР 1. Рассмотрим первую смешанную задачу (1.3)-(1.5), предполагая, что $Q=$ $(0,4 / 3) \times(0,4 / 3), A_{R}=\Delta R_{Q}, R_{Q}=P_{Q} R I_{Q}, R u(x)=u(x)+a u\left(x_{1}+1, x_{2}+1\right)+$ $a u\left(x_{1}-1, x_{2}-1\right), 0<a<1$. Очевидно, разбиение $\mathcal{R}$ области $Q$ состоит из двух классов подобластей: 1$) Q_{11}=(0,1 / 3) \times(0,1 / 3), Q_{12}=(1,4 / 3) \times(1,4 / 3)$ и 2$) Q_{21}=Q \backslash\left(\bar{Q}_{11} \cup\right.$ $\left.\bar{Q}_{12}\right)$. Множество $\mathcal{K}$ принадлежит границе $\partial Q$ и состоит из четырех точек: $g^{1}=(1 / 3,0)$, $g^{2}=(4 / 3,1), g^{3}=(0,1 / 3), g^{4}=(1,4 / 3)$.

Матрицы $A_{s}(x)\left(x \in \bar{Q}_{s 1} ; s=1,2\right)$, определенные по формуле (1.8), имеют вид

$$
\begin{aligned}
& A_{1}(x)=\left(\begin{array}{ll}
1 & a \\
a & 1
\end{array}\right) \quad\left(x \in \bar{Q}_{11}\right), \\
& A_{2}(x)=\left(\begin{array}{ll}
1 & a \\
a & 1
\end{array}\right) \quad\left(x \in \bar{Q}_{21} \cap \mathcal{K}\right), \quad A_{2}(x)=(1) \quad\left(x \in \bar{Q}_{21} \backslash \mathcal{K}\right) .
\end{aligned}
$$

Таким образом, матрицы $A_{s}(x)\left(\xi_{1}^{2}+\xi_{2}^{2}\right)\left(x \in \bar{Q}_{s 1} ; s=1,2\right)$ положительно определены (ср. (1.9)). Следовательно, оператор $-A_{R}-$ сильно эллиптический.

Положим

$$
v_{1}(r, \varphi)=\xi(r) r^{\lambda} \sin \lambda \varphi, \quad v_{2}(r, \varphi)=\xi(r) r^{\lambda} \sin \lambda \varphi\left(\frac{3 \pi}{2}-\varphi\right),
$$


где $\xi(r) \in \dot{C}^{\infty}(\mathbb{R}), \quad 0 \leqslant \xi(r) \leqslant 1, \xi(r)=1$ при $r \leqslant 1 / 8, \xi(r)=0$ при $r \geqslant 1 / 6$, $\lambda=(2 / \pi) \arccos (a / 2), r, \varphi$ - полярные координаты.

Введем функцию $v(x)$ по формуле

$$
v(x)= \begin{cases}\frac{v_{1}\left(x_{1}-1 / 3, x_{2}\right)-a v_{2}\left(x_{1}-1 / 3, x_{2}\right)}{1-a^{2}}, & x \in Q_{11}, \\ \frac{-a v_{1}\left(x_{1}-4 / 3, x_{2}-1\right)+v_{2}\left(x_{1}-4 / 3, x_{2}-1\right)}{1-a^{2}}, & x \in Q_{12}, \\ v_{1}\left(x_{1}-1 / 3, x_{2}\right)+v_{2}\left(x_{1}-4 / 3, x_{2}-1\right), & x \in Q_{21} .\end{cases}
$$

Очевидно,

$$
R_{Q} v(x)=v_{1}\left(x_{1}-\frac{1}{3}, x_{2}\right)+v_{2}\left(x_{1}-\frac{4}{3}, x_{2}-1\right)
$$

Поскольку $0<\lambda<1$, легко видеть, что $v \in \stackrel{\circ}{W} \underset{2}{1},-\Delta R_{Q} v \in L_{2}(Q)$, но $v \notin W_{2}^{2}\left(Q_{s 1} \cap\right.$ $\left.S_{\delta}\left(g^{1}\right)\right)$ для любого $\delta>0$. Следовательно, функция $u(x, t)=t v(x)$ является сильным решением задачи (1.3)-(1.5) для $f(x, t)=v(x)-t \Delta R_{Q} v(x) \in L_{2}\left(\Omega_{T}\right)$ и $\varphi(x)=0$. Однако, $u \notin W_{2}^{2,0}\left(\left(Q_{s 1} \cap S_{\delta}\left(g^{1}\right)\right) \times(0, T)\right)$ для любого $\delta>0$. Таким образом, при $\varepsilon=0$ теорема 5 , вообше говоря, не верна.

Покажем теперь, что

$$
\left.u_{x_{1}}\right|_{x_{1}=1 / 3+0, x_{2} \leqslant 1 / 8} \neq\left. u_{x_{1}}\right|_{x_{1}=1 / 3-0, x_{2} \leqslant 1 / 8} .
$$

В силу (4.4) для этого достаточно убедиться, что

$$
\left.v_{1 \varphi}\right|_{\varphi=\pi / 2, r \leqslant 1 / 8} \neq \frac{1}{1-a^{2}}\left\{\left.v_{1 \varphi}\right|_{\varphi=\pi / 2, r \leqslant 1 / 8}-\left.a v_{2 \varphi}\right|_{\varphi=\pi / 2, r \leqslant 1 / 8}\right\} .
$$

Соотношение (4.5) эквивалентно следующему

$$
\lambda \cos \frac{\lambda \pi}{2} \neq \frac{1}{1-a^{2}}\left(\lambda \cos \lambda \frac{\pi}{2}-\lambda \cos \lambda \pi\right) .
$$

Приводя подобные и используя равенство $\cos (\lambda \pi / 2)=a / 2$, мы можем переписать (4.6) в виде

$$
a^{3}-a^{2}+2 \neq 0
$$

Поскольку корни уравнения $a^{3}-a^{2}+2=0$ имеют вид $a_{1,2}=1 \pm i, a_{3}=-1$, условие (4.7) выполняется при $0<a<1$. Поэтому $u \notin W_{2}^{2,0}\left(S_{\sigma}(y)\right)$ для любых $y=\left(y_{1}, y_{2}\right)$ и $\sigma>0$ таких, что $y_{1}=1 / 3,0<y_{2}<1 / 8, \sigma<y_{2}$. Таким образом, гладкость сильных решений задачи (1.3)-(1.5) может нарушаться на гранище соседних подобластей $Q_{s_{1} l_{1}} \times(0, T)$ и $Q_{s_{2} l_{2}} \times(0, T)$. 


\section{СПИСОК ЦИТИРОВАННОЙ ЛИТЕРАТУРЫ}

[1] Vorontsov M.A., Ricklin J. C., Carhart G.W. Optical simulation of phase-distorted imaging systems: nonlinear and adaptive optics approach // Optical Engineering. 1995. V. 34. № 11. P. 3229-3238.

[2] Скубачевский А. Л. О некоторых свойствах эллиптических и параболических функционално-дифференциалных уравнений // УМН. 1996. Т. 51. №1. С. 169-170.

[3] Skubachevskii A. L. Bifurcation of periodic solutions for nonlinear parabolic functional differential equations arising in optoelectronics // Nonlinear Anal. 1998. V. 32. № 2. P. 261-278.

[4] Лионс ЖК.-Л., Мадженес Э. Неоднородные граничные задачи и их приложения. М.: Мир, 1971.

[5] Skubachevskii A. L. Elliptic Functional Differential Equations and Applications. Basel-Boston-Berlin: Birkhauser, 1997.

[6] Pazy A. Semigroups of Linear Operators and Applications to Partial Differential Equations. New York-Berlin-Heidelberg: Springer, 1983.

[7] Иосида К. Функциональный анализ. М.: Мир, 1967.

Московский государственный авиационный институт

Поступило 20.07 .98 